\title{
Utility of computed tomography and derivation and validation of a score to identify an emergent outcome in 2,315 patients with suspected urinary tract stone
}

\author{
F. Kris Aubrey-Bassler, MD, MSc*; Scott D. Lee, BSc, MSc(c) ${ }^{\dagger}$; Richard B. Barter, MD, LRCP ; \\ Shabnam Asghari, MD, $\mathrm{PhD}^{\ddagger \S}$; Richard Cullen, $\mathrm{BSc}, \mathrm{MSc}(\mathrm{c})^{* \S}$; Marshall Godwin, MD, MSc ${ }^{\S}$
}

\begin{abstract}
Objective: Because a majority of urinary tract stones (UTSs) pass spontaneously and clinically significant alternative pathology is rare, we hypothesize that many computed tomographic (CT) scans to diagnose them are likely unnecessary. We sought to measure the impact of renal CT scans on resource use and to justify a prospective study to derive a score that predicts an emergent diagnosis in patients with suspected UTS by doing so in our retrospective series.

Methods: We conducted a retrospective study of ED patients who had noncontrast CT of the abdomen for suspected UTS. A split-sample was used to derive and validate a score to predict the presence of an emergent diagnosis on CT.

Results: Of the 2,315 patients $(50.8 \%$ female, mean age 45 years), $49(2.1 \%)$ had an emergent outcome observed on CT. An additional $12(0.5 \%)$ patients had an urgent outcome and $239(10.6 \%)$ had a urologic procedure within 8 weeks of the CT. Serum white blood cell count, highest temperature, urine red blood cell count, and the presence of abdominal pain were significant predictors of the primary outcome. A score derived using these predictors had a potential range of -2 $(0.26 \%$ predicted risk, $0.5 \%$ actual risk of the outcome) to 6 (52\% predicted risk). The score was moderately discriminatory with c-statistics of 0.752 (derivation) and 0.668 (validation) and accurate with Hosmer-Lemeshow statistics of 10.553 ( $p=0.228$, derivation) and 9.70 ( $p=0.286$, validation). Conclusions: A sensible, relevant score derived and validated on all patients presenting with symptoms suggestive of renal colic could be useful in reducing abdominal CT scan ordering.
\end{abstract}

\section{RÉSUMÉ}

Objectifs: Comme la plupart des calculs rénaux (CR) sont évacués spontanément et que le nombre d'autres affections possibles cliniquement importantes est très faible, nous avons émis I'hypothèse selon laquelle bon nombre des tomodensitométries (TDM) effectuées pour faciliter la pose du diagnostic sont probablement inutiles. Nous avons cherché à mesurer l'incidence des TDM rénales sur I'utilisation des ressources et à justifier la pertinence d'une étude prospective afin de dériver un résultat qui permettrait de poser un diagnostic prévisionnel d'affection nécessitant une intervention immédiate chez des patients chez qui l'on soupçonnait la présence de $\mathrm{CR}$, et ce, en faisant de même dans notre série rétrospective de cas.

Méthode: Nous avons mené une étude rétrospective sur des patients examinés au service des urgences, qui avaient subi une TDM abdominale sans injection de produit de contraste pour une évaluation de la présence présumée de CR. Nous avons fractionné un échantillon pour dériver et valider un résultat permettant de poser un diagnostic prévisionnel d'affection nécessitant une intervention immédiate à partir des images obtenues à la TDM.

Résultats: Sur les 2,315 patients (femmes: $50.8 \%$; âge moyen: 45 ans), $49(2.1 \%)$ ont obtenu, à la TDM, un résultat nécessitant une intervention immédiate; 12 autres patients $(0.5 \%)$ ont obtenu un résultat nécessitant une intervention urgente et 239 patients $(10.6 \%)$ ont subi une intervention urologique dans les 8 semaines suivant la TDM. Le nombre de globules blancs dans le sang, une température élevée, le nombre de globules rouges dans l'urine, et la présence de douleur abdominale étaient des variables prévisionnelles importantes du principal critère d'évaluation. Le résultat dérivé de ces variables prévisionnelles donnait une plage possible variant de -2 (risque prévu: $0.26 \%$; risque réel: $0.5 \%$ ) à 6 (risque prévu: $52 \%$ ). Le résultat était modérément discriminatoire $d^{\prime}$ après la statistique $C$, qui avait des valeurs de 0.752 (dérivation) et de 0.668 (validation), et précise d'après le test d'Hosmer-Lemeshow, qui avait des valeurs

From the ${ }^{*}$ Centre for Rural Health Studies, Faculty of Medicine, Memorial University, St. John's, NL; †Faculty of Medicine, Dalhousie University, Halifax, NS; łFaculty of Medicine, Memorial University, St. John's, NL; and §Primary Healthcare Research Unit, Faculty of Medicine, Memorial University, St. John's, NL.

Correspondence to: Dr. Kris Aubrey-Bassler, Primary Healthcare Research Unit, Memorial University of Newfoundland, Agnes Cowan Hostel, Room 427, 300 Prince Philip Drive, St. John's, NL A1B 3V6; kaubrey@mun.ca.

This article has been peer reviewed. 
de 10.553 ( $p=0.228$, dérivation) et de $9.70(p=0.286$, validation).

Conclusions: Un résultat sensible, pertinent, dérivé de données existantes, puis validé sur tous les patients présentant des symptômes évocateurs d'une colique néphrétique pourrait se révéler utile dans la diminution du nombre de demandes de TDM abdominales.

Keywords: diagnostic errors, decision support techniques, emergency medicine, nephrolithiasis, $x$-ray computed tomography
The authors of a recent meta-analysis estimated that $47 \%$ of urinary tract stones 6 to $10 \mathrm{~mm}$ and $68 \%$ of urinary tract stones $5 \mathrm{~mm}$ or smaller in diameter will pass spontaneously without medical intervention and without long-term sequelae. ${ }^{1}$ Given that stone passage can be delayed, many patients suffering from stones will have had a computed tomographic (CT) scan to confirm the diagnosis and rule out other conditions. Although CT scans are highly sensitive for nephrolithiasis and help clarify alternative diagnoses, they are also costly and time-consuming to perform and expose patients to potentially unnecessary radiation. ${ }^{2}$

We and others ${ }^{3}$ hypothesized that a significant proportion of patients with suspected urinary tract stones can be safely treated without a CT scan and that these patients can be reliably identified based on characteristics observed at their initial presentation to the emergency department (ED). Although a recent review article ${ }^{4}$ and summary literature ${ }^{5}$ also appear to agree, they do not specify which symptoms and signs can be used for this purpose. These references also highlight some previous literature that appears to show a high rate of significant alternative diagnosis on CT scans ordered for suspected renal colic, ${ }^{6-8}$ information that clinicians likely use to justify CT scans that might not have been ordered otherwise. We conducted this retrospective observational study of patients who had a renal protocol CT scan to further explore these issues and to see if a similar prospective study was justified.

Clinical experience informed us that the most useful rule to use in patients with suspected renal colic would be one that identifies patients having emergent (requiring immediate intervention) and urgent pathology (requiring subacute intervention). However, interim analyses on half of the data collected for this study using recursive partitioning, logistic regression and correlation, and regression tree classification indicated that such a rule would not be possible, nor was it possible to derive a sufficiently sensitive and specific rule that specified a distinct course of action (i.e., ordering a CT scan) at a defined score cutpoint. Therefore, we focused on deriving a score that would calculate the risk of a patient having an emergent diagnosis who required intervention guided by CT results at the initial ED visit. We hypothesize that the use of such a tool derived and validated prospectively on all patients presenting with symptoms suggestive of renal colic will translate into reduced use of $\mathrm{CT}$.

\section{METHODS}

\section{Study design and setting}

We retrospectively accessed the charts of all patients who had an unenhanced CT scan of the abdomen and pelvis ordered by a physician practicing in the two adult EDs of our multisite academic hospital (Health Sciences Centre and St. Clare's Mercy Hospital) during the calendar years 2005 to 2008 inclusive. The renal colic literature has not substantively changed its recommendations on CT use for this purpose since that time, ${ }^{4,5}$ nor have our internal departmental policies and procedures changed.

Annual visits at these two EDs total approximately 90,000 . It is the primary hospital for a local catchment of 188,000 and the only tertiary care hospital in this isolated Canadian province of 505,000 people. ${ }^{9}$ Access to the island portion of the province is exclusively by air or 5- to 6-hour ocean-going ferry. During the study period, all of the urologists in the province practiced at one of the sites of our academic institution, and their procedure records are catalogued in the electronic medical record system used for this study. Thus, we can be more confident of the thoroughness of our follow-up than a retrospective design might elsewhere permit. The study was approved by the Human Investigations Committee of Memorial University.

\section{Selection of participants}

We excluded patients who were less than 18 years old, transferred from a peripheral hospital, or had a previous solitary kidney or renal transplant documented on their ED visit record. Patients with other previous kidney disease were included if they had a normal serum 
creatinine. An elevated creatinine documented at any time in the 7 days prior to the ED visit was grounds for exclusion, as was an abdominal surgical procedure within 14 days prior to presentation. Patients who had a contrast-enhanced CT scan of the abdomen performed on the same day as the renal protocol scan were also excluded unless the contrast-enhanced scan was ordered for the further characterization of abnormalities on the initial renal protocol CT scan.

\section{Data collection and processing}

Data potentially predictive of outcomes (Table 1) were accessed from the electronic hospital record. We also accessed the radiologist's CT interpretation and, for those patients without a definitive CT diagnosis, the results of subsequent investigations to clarify it. Finally, we documented the need for urologic procedure or hospital admission related to the initial presentation in the 8-week period following the initial ED visit. Any one of a dictated procedure note, a visit to a urologist in surgical day care recorded in the visit history of the electronic record, or the report of a compatible fluoroscopic procedure was considered sufficient to indicate a urologic procedure.
All of the data in the current study were collected by a single abstractor, who underwent specific training under the supervision of the first author. Data were entered into a standardized electronic abstraction form. The primary investigator verified all data for the first 50 patients, and data for the subsequent 50 patients were closely scrutinized. After this detailed data review, the data abstractor flagged any uncertainties and reviewed them with the primary investigator. In addition, screening for outliers and out-of-range values was conducted on the remaining data and discrepancies were adjusted with a full chart review by the abstractor and/or the primary author if necessary.

\section{Outcome measures}

CT findings were classified according to both the presence of stone and other novel (i.e., not known previously) pathology. These categories included 1) emergent (requiring immediate clinical intervention); 2) urgent (for which a delay in diagnosis of several days was unlikely to affect outcomes); 3) probable benign pathology for which no record of appropriate followup was available; 4) probable benign pathology for

\begin{tabular}{|c|c|c|c|c|c|}
\hline \multirow[b]{2}{*}{ Characteristic } & \multirow[b]{2}{*}{$n$} & \multicolumn{2}{|c|}{$\begin{array}{c}\text { Data for patients with and without } \\
\text { emergent outcome }\end{array}$} & \multicolumn{2}{|c|}{ Logistic regression parameters } \\
\hline & & Without & With & $\beta$ & $p$ \\
\hline \multicolumn{6}{|l|}{ Demographic } \\
\hline Age, mean (SD) & 2,315 & $45.0(14.3)$ & $46.1(15.0)$ & 0.006 & 0.578 \\
\hline Female, $n(\%)$ & 1,177 & $1,149(97.6)$ & $28(2.4)$ & 0.361 & 0.229 \\
\hline Male, $n(\%)$ & 1,138 & $1,119(98.3)$ & $19(1.7)$ & Ref & \\
\hline \multicolumn{6}{|l|}{ Clinical } \\
\hline CTAS score, mode (range) & 2,314 & $3(2-5)$ & $3(2-5)$ & 0.084 & 0.726 \\
\hline History of stone, $n(\%)$ & 2,315 & $39 / 1,540(2.5)$ & $8 / 775(1.0)$ & -0.913 & 0.019 \\
\hline Abdominal pain, $n(\%)$ & 2,315 & $21 / 1,486(1.4)$ & $26 / 829(3.1)$ & 0.815 & 0.006 \\
\hline Groin pain, $n(\%)$ & 2,315 & $40 / 2,025(2.0)$ & $7 / 290(2.4)$ & 0.205 & 0.621 \\
\hline Flank pain, $n(\%)$ & 2,315 & 26/926 (2.8) & 21/1,389 (1.5) & -0.632 & 0.033 \\
\hline Highest temperature: ${ }^{\circ} \mathrm{C}$, mean (SD) & 2,255 & $36.6(0.5)$ & $37.1(0.9)$ & 0.150 & 0.025 \\
\hline Lowest SBP: mm Hg, mean (SD) & 2,267 & $126(21.5)$ & $123(21.2)$ & -0.009 & 0.234 \\
\hline Highest HR, mean (SD) & 2,287 & $83.9(14.8)$ & $93.0(20.0)$ & 0.037 & $<0.001$ \\
\hline \multicolumn{6}{|l|}{ Laboratory } \\
\hline Serum WBC: $\times 10^{9} / \mathrm{L}$, mean $(S D)$ & 1,865 & $9.75(3.24)$ & $12.17(3.79)$ & 0.198 & $<0.001$ \\
\hline Serum creatinine: $\mathrm{mmol} / \mathrm{L}$, mean (SD) & 1,805 & $83.3(17.0)$ & $83.9(16.8)$ & 0.001 & 0.955 \\
\hline Urine $\mathrm{RBC}$, mode (range) & 2,123 & $4+($ neg-4+) & Trace (neg-4+) & 0.237 & 0.017 \\
\hline Urine WBC, mode (range) & 2,123 & Negative (neg-4+) & Negative (neg-2+) & -0.064 & 0.277 \\
\hline Urine nitrites positive, $n(\%)$ & 2,124 & $39 / 2,003(1.9)$ & 2/112 (1.8) & -17.284 & 0.999 \\
\hline
\end{tabular}


which a record of appropriate follow-up was not available but medical visits were made more than 1 year later with no mention of related pathology; 5) benign pathology; and 6) no pathology found. This classification was performed independently by two emergency physicians who were blinded to all other information. Agreement was measured using the kappa statistic. Differences were resolved by consensus and when necessary by discussion with a third emergency physician. The presence of emergent pathology on a CT scan was our primary outcome of interest. To provide an estimate of utility, we assumed that CT scans demonstrating emergent or urgent results or stones requiring urologic intervention within 8 weeks of the ED visit influenced patient care.

\section{Primary data analysis}

The best linear or nonlinear relationship between predictor variables and outcomes was determined with fractional polynomial functions using the "MFP" command in Stata version 11.2 (StataCorp., College Station, TX). ${ }^{10} \mathrm{We}$ measured the bivariate association between candidate predictor variables and the outcome variable using logistic regression. Predictors that were significant at the $p<0.10$ level were then entered into a multivariate backwards stepwise logistic regression model with significance set to $p<0.05$ for variable retention.

The methods of Sullivan and colleagues ${ }^{11}$ were then used to modify the results of the logistic model into a risk score. We chose the increase in risk of our outcome associated with a $1^{\circ}$ increase in temperature as the risk attributed to 1 point in the score. Seventy percent of the records were randomly selected as a derivation data set, and the remaining 30\% were used as a validation set for the score. We measured the performance of our score using the c-statistic and the Hosmer-Lemeshow goodness-of-fit test. ${ }^{12}$ The c-statistic is equivalent to the probability of a case having a higher score result than a noncase (discrimination), and the Hosmer-Lemeshow test is a measure of how close the risk predicted by the test score is to the actual risk of the disease of interest in the studied population (calibration).

\section{RESULTS}

Figure 1 outlines the steps to screen patient records for inclusion and exclusion from the study. Patient characteristics are outlined in Table 1. Initial interrater reliability between the two investigators classifying the final diagnoses was $\kappa=0.90$ (95\% CI $0.88-0.91 ; p<$ 0.001 ), which increased to $\kappa=0.99$ (95\% CI 0.99 $0.99 ; p<0.001)$ after discussion of the discrepancies. At this stage, agreement on the urgent and emergent categorizations was $100 \%$. A third emergency physician reviewed the CT reports of the 46 remaining patients on whom the original two reviewers did not initially agree. All of these patients were ranked in adjacent categories by the two initial physicians, and none had either urgent or emergent diagnoses.

The CT findings of patients from different categories are presented in Table 2 and Table 3. An additional 674 patients had benign CT findings such as ovarian, renal, and hepatic cysts; vascular and prostatic calcification; nonobstructing gallstones; and congenital abnormalities of the kidney and benign tumours of the ovaries, uterus, kidneys, liver, and adrenal glands (not a complete list). Nine patients were found to have previously undiagnosed abdominal aortic aneurysms that were not leaking on the initial assessment and did not require surgical management during the follow-up period; these patients were included in the benign category.

A cross-classification of patients according to the presence of a stone and other pathology is presented in Table 4. For the 16 patients with evidence of a stone and an emergent diagnosis, 7 had CT evidence of pyelonephritis and 2 had ruptured calyces. These findings were likely secondary to the observed stone. Of the remaining patients with both a stone and an emergent $\mathrm{CT}$ diagnosis, two had images compatible with aortic dissection, three had diverticulitis, and two had pancreatitis. Lymphoma, prostatic intraepithelial neoplasia, and renal cell carcinoma were the other diagnoses observed in three patients with both an urgent diagnosis and stones.

Figure 2 demonstrates the incidence of hospitalization and urologic procedure following the index ED visit. Using our pre-specified definition of CT utility, in addition to the patients with emergent and urgent diagnoses, an additional 246 patients had a urologic procedure within 8 weeks, for a total of 307 (13.3\%) patients.

Bivariate association data between all independent variables and an emergent outcome are presented in Table 1. Transformation of the continuous variables did not result in a significant improvement to model fit, and all variables were therefore modeled using linear 


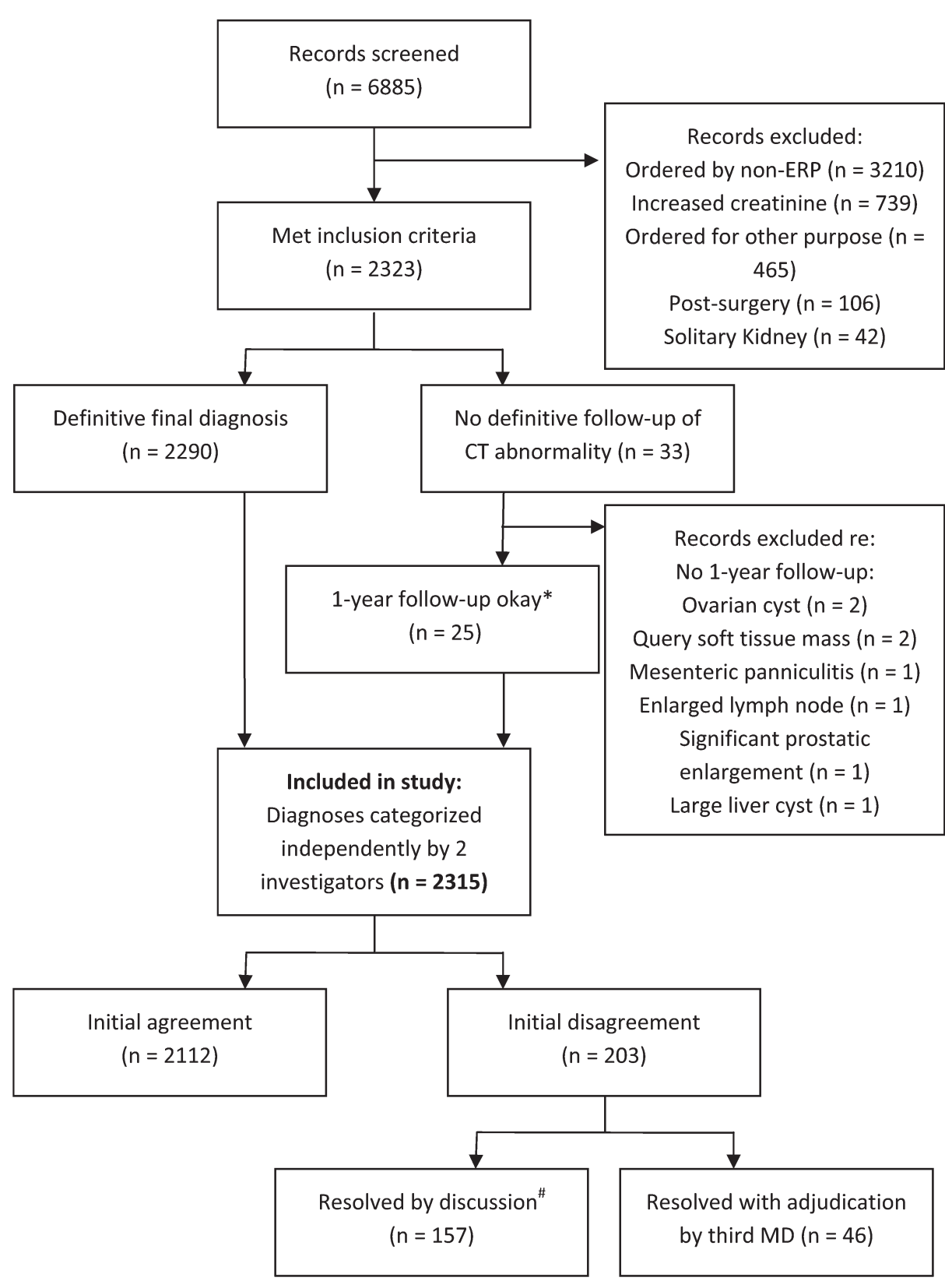

Figure 1. Flow diagram for patients considered for enrolment in trial. *A chart record from at least 1 year after computed tomography (CT) was present and made no mention of pathology attributable to the abnormality on the initial CT scan. ${ }^{\text {A Agreement was }}$ $100 \%$ for urgent and emergent categories prior to adjudication by the third physician. ERP = emergency room physician. functions. Of the 2,315 patients, 1,611 (69.6\%) had complete data for all four significant predictors (Table 5), and $70 \%$ of these were included in derivation $(n=1128)$ and $30 \%$ in validation $(n=483)$ of the score. Interpolation of missing data using various techniques did not significantly alter model parameters (data not shown). Scores allotted to variables are presented in Table 6. The WATUR (white blood cell count, abdominal pain, temperature, unrine red blood cell count) score (Figure 3) was moderately discriminatory with cstatistics of 0.752 (95\% CI 0.651-0.853) and 0.668 (95\% $0.445-0.891)$ for the derivation and validation data sets, respectively. The c-statistic for the score using all data was 0.761 (95\% CI 0.678-0.844), indicating moderate discrimination. The Hosmer-Lemeshow statistic for the derivation data set was $10.553(d f=8, p=0.228)$ and for the validation data set was $9.70(d f=8, p=0.286)$, suggesting that the model fits the data reasonably well.

\section{DISCUSSION}

As we hypothesized, we have documented low rates of CT findings that may have altered patient management $(13.3 \%)$ and a high rate of CT scans for which 


\begin{tabular}{|c|c|}
\hline Diagnosis & $n(\%)$ \\
\hline Ovarian cyst or follicle & $11(0.5)$ \\
\hline Mesenteric panniculitis & $6(0.3)$ \\
\hline Renal cyst & $2(0.1)$ \\
\hline Query soft tissue mass & $2(0.1)$ \\
\hline Sacroiliitis & $1(0.04)$ \\
\hline Query lytic lumbar spine lesion & $1(0.04)$ \\
\hline Query solid renal mass & $1(0.04)$ \\
\hline Query solid liver mass & $1(0.04)$ \\
\hline Total & $25(1.1)$ \\
\hline
\end{tabular}

no pathology was observed ( $n=501,21.6 \%$; see Table 4). The $2.6 \%$ rate of "clinically significant pathology" other than urinary tract stones that we have observed here is significantly lower than the $14.4 \%^{6}$ and $18 \%^{7}$ rates reported elsewhere. The higher rates reported in those studies have been used to recommend the routine use of CT for the diagnosis

\begin{tabular}{|c|c|c|}
\hline $\begin{array}{l}\text { Pathology } \\
\text { classification }\end{array}$ & Final diagnosis & $\begin{array}{c}\text { Patients, } \\
n(\%)\end{array}$ \\
\hline \multirow[t]{15}{*}{ Emergent } & Diverticulitis & $17(0.7)$ \\
\hline & Pyelonephritis & $9(0.4)$ \\
\hline & Appendicitis & $6(0.3)$ \\
\hline & Pancreatitis & $3(0.1)$ \\
\hline & Aortic dissection & $2(0.1)$ \\
\hline & Bowel perforation & $2(0.1)$ \\
\hline & Cholecystitis & $2(0.1)$ \\
\hline & Ruptured renal calyx & $2(0.1)$ \\
\hline & Bowel obstruction & $1(0.04)$ \\
\hline & Pelvic inflammatory disease & $1(0.04)$ \\
\hline & Pneumonia & $1(0.04)$ \\
\hline & Prostatitis with fever & $1(0.04)$ \\
\hline & Renal abscess & $1(0.04)$ \\
\hline & RCC with large hemorrhage & $1(0.04)$ \\
\hline & Total & $49(2.1)$ \\
\hline \multirow[t]{8}{*}{ Urgent } & Renal cell cancer & $5(0.2)$ \\
\hline & Bladder cancer & $2(0.1)$ \\
\hline & Cancer unknown primary & $1(0.04)$ \\
\hline & Lymphoma & $1(0.04)$ \\
\hline & Ovarian cancer & $1(0.04)$ \\
\hline & Pancreatic cancer & $1(0.04)$ \\
\hline & Prostatic intraepithelial neoplasia & $1(0.04)$ \\
\hline & Total & $12(0.5)$ \\
\hline
\end{tabular}

Table 4. Presence of stone cross-classified with other diagnosis category

\begin{tabular}{lcc} 
& \multicolumn{2}{c}{ Presence of stone, $n(\%)$} \\
\cline { 2 - 3 } $\begin{array}{l}\text { Other diagnosis } \\
\text { category }\end{array}$ & Yes & No \\
Emergent diagnosis & $16(0.9)$ & $33(1.4)$ \\
Urgent diagnosis & $3(0.1)$ & $9(0.4)$ \\
Benign diagnosis & $424(18.3)$ & $300(13.0)$ \\
None & $1,029(44.4)$ & $501(21.6)$ \\
Total & $1,472(63.6)$ & $843(36.4)$
\end{tabular}

of suspected renal colic; however, their definition of significant pathology appears to include that requiring further workup such as ultrasonography or follow-up CT. We found a similar $13.1 \%$ rate of "significant pathology" using that definition (data not shown). In our series, however, most of these follow-up tests revealed benign pathology with no lasting effect on morbidity; we have therefore not included these patients in this category. In comparison, other research has found a $5.9 \%$ rate of other significant pathology. ${ }^{13}$ Although the definition of significant pathology used in that study is more inclusive than ours, the inclusion of follow-up test results permitted us to apply a definition similar to ours, resulting in a significant pathology rate of $3.3 \%$, similar to the $2.6 \%$ rate observed here. In a similar fashion, we were able to estimate a rate of $4.5 \%$ in a large series of 1,000 patients. ${ }^{8}$ The rates of significant alternate pathology of $4 \%^{14}$ and $6 \%^{15}$ in two relatively small series are remarkably consistent. A relatively low rate of significant pathology increases the likelihood that we will be able to successfully derive a clinical decision rule.

Our study documented a stone in $63.6 \%$ of the patients enrolled. This is remarkably consistent with the $61.9 \%,{ }^{13} 49.5 \%,{ }^{16} 58 \%,{ }^{3} 57.6 \%,{ }^{6} 60 \%,{ }^{7} 66 \%,{ }^{15}$ and $63 \%^{14}$ rates observed in other studies despite slight differences in enrolment criteria. This consistency suggests that the ED ordering practices for suspected renal colic are quite similar across facilities and increases the likelihood that our results will generalize to other settings; however, this hypothesis will require further verification.

It is possible that a reduction in the use of $\mathrm{CT}$ at the index ED visit will increase the rate of repeat patient visits to the ED because of diagnostic uncertainty. Gottlieb and colleagues compared patients with 


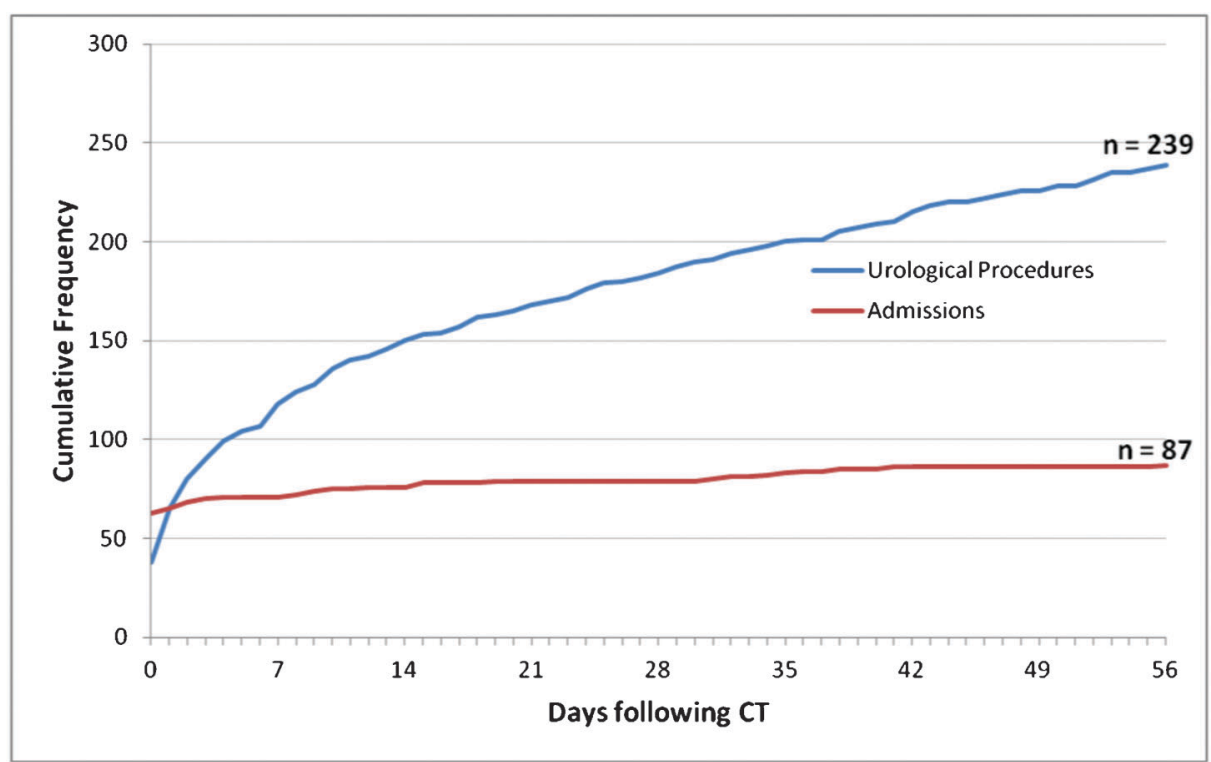

Figure 2. Cumulative number of admissions and urologic procedures among the patient sample for 8 weeks following computed tomography (CT). suspected renal colic between two 1-year periods, the first with relatively low $(8.6 \%)$ and the second with relatively high $(90.0 \%)$ use of CT. ${ }^{13}$ The rate of hospital admission directly from the ED was essentially identical during these two periods, and no significant increase in either subsequent hospital admission or return ED visits during the low CT use period was observed. These findings suggest that tools designed to reduce CT use will have a neutral effect on subsequent ED use and hospital admission.

\section{LIMITATIONS}

As an estimate of the clinical utility of CT in these patients, we included only those patients having urologic procedures within 8 weeks of the CT study. Whereas some patients had nonurologic procedures (e.g., for ovarian cysts, total $n=57$ ) based on the findings of the CT, other patients had urologic procedures after the 8-week study period or repeat CT scans (on which the decision to conduct a procedure was made) prior to having a procedure

\begin{tabular}{|c|c|c|c|}
\hline & Odds ratio & $95 \% \mathrm{Cl}$ & $p$ \\
\hline Serum WBC & 1.20 & $1.08-1.34$ & 0.001 \\
\hline Urine RBC dipstick & 0.750 & $0.58-0.98$ & 0.034 \\
\hline Temperature & 2.63 & $1.59-4.36$ & $<0.001$ \\
\hline Abdominal pain & 3.44 & $1.50-7.88$ & 0.003 \\
\hline
\end{tabular}

within the 8-week period. Thus, this "utility" metric should be interpreted with this information in mind. It would have been interesting to collect and analyze data on all procedures conducted within the study period.

Because of the geographic isolation of our study population, there is little opportunity to obtain urologic care outside of our institution. Thus, we can be more certain of the thoroughness of the follow-up than this study design would normally permit. We have not confirmed whether some patients obtained urologic care outside of our province but expect that this would be a very small number.

\begin{tabular}{|c|c|c|}
\hline & Value & Score \\
\hline \multirow[t]{5}{*}{ WBC $\left(\times 10^{9} / L\right)$} & $<4$ & 0 \\
\hline & $4.1-12$ & 0 \\
\hline & $12.1-15$ & 1 \\
\hline & $15.1-18$ & 2 \\
\hline & $>18$ & 3 \\
\hline \multirow[t]{3}{*}{ Urine RBC } & None-trace & 0 \\
\hline & $1+-2+$ & -1 \\
\hline & $3+$-gross & -2 \\
\hline \multirow[t]{4}{*}{ Temperature $\left({ }^{\circ} \mathrm{C}\right)$} & $<35$ & 0 \\
\hline & $35-37.9$ & 0 \\
\hline & $38.0-38.5$ & 1 \\
\hline & $>38.5$ & 2 \\
\hline \multirow[t]{2}{*}{ Abdominal pain } & No & 0 \\
\hline & Yes & 1 \\
\hline
\end{tabular}




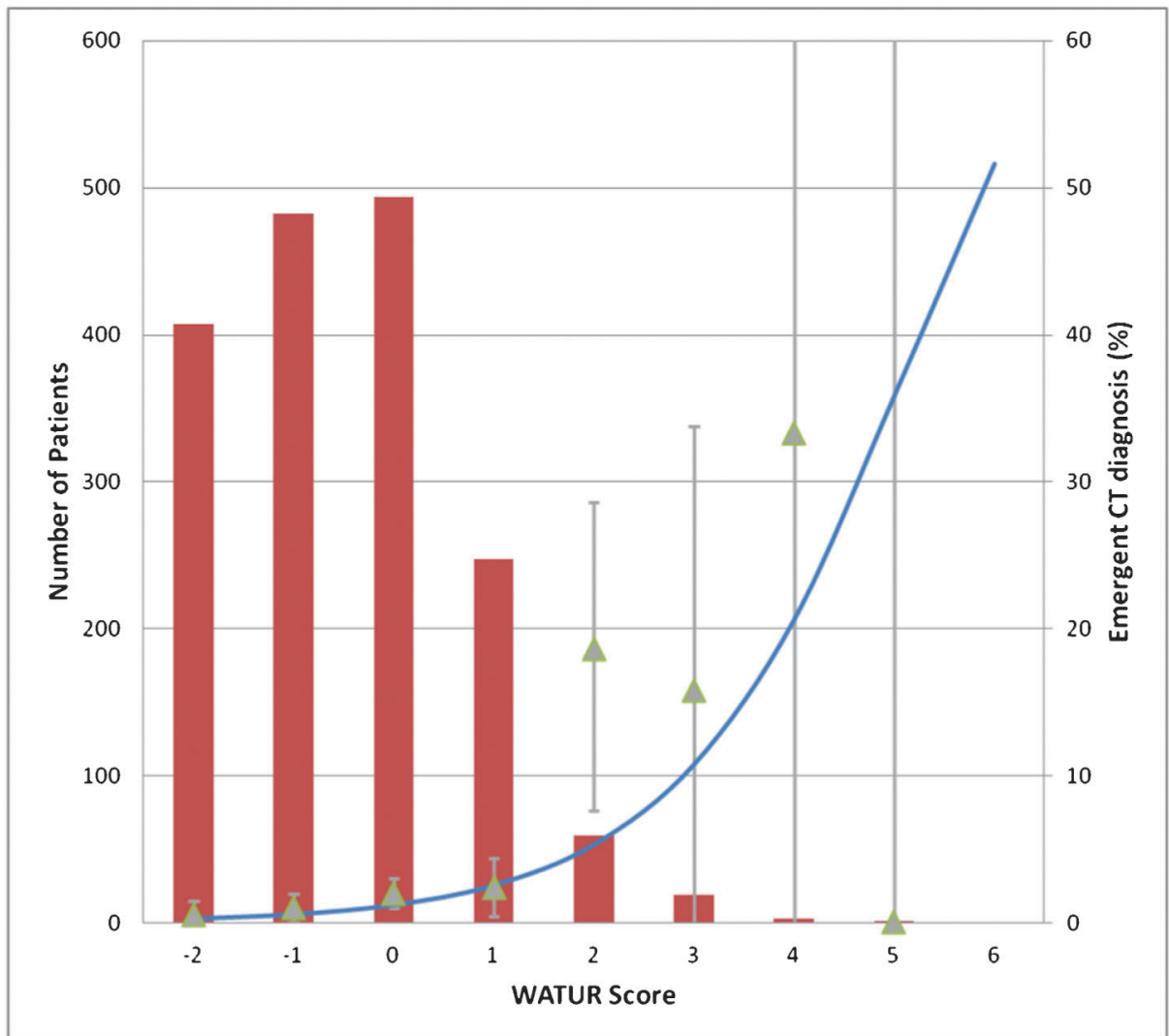

Figure 3. Performance of the WATUR (white blood cell count, abdominal pain, temperature, ürine red blood cell count) score for an emergent diagnosis in patients having a renal protocol computed tomographic (CT) scan. Bars represent the number of patients at each score (left vertical axis). Triangles represent the actual risk of an emergent CT diagnosis at a given score (right vertical axis), and the error bars represent the $95 \%$ confidence interval. The blue line is the calculated regression line.

Our electronic hospital record does not allow us to reliably identify patients who presented to the ED with suspected renal colic yet did not have a CT scan. Therefore, we conducted our study only on patients who could be identified through their renal protocol CT record because it was within our means and because we believed it would be required to justify funding for a more inclusive trial. Clearly, before this score is put into widespread use, it will need to be at least validated, if not rederived, on a population including all patients presenting with suspected stone.

\section{CONCLUSIONS}

We have demonstrated that a high proportion of renal protocol CT scans do not appear to have impacted patient care and have derived a score based on clinical and demographic data to predict an emergent diagnosis on the CT scan. This category of diagnosis is the most important to elucidate on the initial ED visit, and other potential causes of symptoms can wait safely for reevaluation if symptoms persist for a period of time or if other alarm symptoms develop (e.g., fever, anuria, intractable pain). These results demonstrate the feasibility of a prospective study to derive and validate a rule on all patients presenting with suspected renal colic. We are currently conducting such a study and will also collect data to help us determine the appropriate length of time with persistent symptoms after which further investigation is warranted, based on the usual time that stones take to pass, and any increased risk of complication associated with delay. It remains to be seen whether this rule will influence clinician CT ordering practices.

Acknowledgements: We wish to thank Drs. Gena Bugden and Melanie Belisle, Ms. Heather O'Reilly, and Mr. Peter Bazeley for assistance with data collection for earlier versions of this work.

Competing interests: This work was funded by the Newfoundland and Labrador Centre for Applied Health Research and The Health Care Foundation of Eastern Health.

\section{REFERENCES}

1. Preminger GM, Tiselius H, Assimos DG, et al. 2007 guideline for the management of ureteral calculi. 7 Urol 2007;178:2418-34, doi:10.1016/j.juro.2007.09.107. 
2. Brenner DJ, Hall EJ. Computed tomography—an increasing source of radiation exposure. N Engl f Med 2007;357:227784, doi:10.1056/NEJMra072149.

3. Broder J, Bowen J, Lohr J, et al. Cumulative CT exposures in emergency department patients evaluated for suspected renal colic. 7 Emerg Med 2007;33:161-8, doi:10.1016/ j.jemermed.2006.12.035.

4. Graham A, Luber S, Wolfson AB. Urolithiasis in the emergency department. Emerg Med Clin North Am 2011;29: 519-38, doi:10.1016/j.emc.2011.04.007.

5. Curhan GC, Aronson MD, Preminger GM. Diagnosis and acute management of suspected nephrolithiasis in adults. In: Goldfarb S, O'Leary MP, editors. UpToDate. 20.7th ed. Waltham (MA): UpToDate; 2012.

6. Ha M, MacDonald RD. Impact of CT scan in patients with first episode of suspected nephrolithiasis. 7 Emerg Med 2004; 27:225-31, doi:10.1016/j.jemermed.2004.04.009.

7. Abramson S, Walders N, Applegate KE, Gilkeson RC, et al. Impact in the emergency department of unenhanced CT on diagnostic confidence and therapeutic efficacy in patients with suspected renal colic: a prospective survey. $A \mathcal{F} R A m \mathcal{F}$ Roentgenol 2000;175:1689-95.

8. Katz DS, Scheer M, Lumerman JH, et al. Alternative or additional diagnoses on unenhanced helical computed tomography for suspected renal colic: experience with 1000 consecutive examinations. Urology 2000;56:53-7, doi:10. 1016/S0090-4295(00)00584-7.

9. Population and dwelling counts, for Canada, provinces and territories, 2006 and 2001 censuses - 100\% data. 2010. Available at: http://www12.statcan.gc.ca/census-recensement/
2006/dp-pd/hlt/97-550/Index.cfm?TPL =P1C\&Page =RETR $\& \mathrm{LANG}=$ Eng\&T $=101$ (accessed January 15, 2012).

10. Sauerbrei W, Meier-Hirmer C, Benner A, et al. Multivariable regression model building by using fractional polynomials: description of SAS, Stata and R programs. Comput Stat Data Anal 2006;50:3464-85, doi:10.1016/ j.csda.2005.07.015.

11. Sullivan LM, Massaro JM, D’Agostino RB Sr. Presentation of multivariate data for clinical use: the Framingham study risk score functions. Stat Med 2004;23:1631-60, doi:10.1002/ $\underline{\operatorname{sim} .1742}$.

12. Harrell FEJ. Regression modeling strategies. New York City: Springer-Verlag; 2001.

13. Gottlieb RH, La TC, Erturk EN, et al. CT in detecting urinary tract calculi: influence on patient imaging and clinical outcomes. Radiology 2002;225:441-9, doi:10.1148/ radiol.2252020101.

14. Poletti P, Platon A, Rutschmann OT, et al. Low-dose versus standard-dose CT protocol in patients with clinically suspected renal colic. A7R Am 7 Roentgenol 2007;188:92733, doi:10.2214/AJR.06.0793.

15. Heneghan JP, McGuire KA, Leder RA, et al. Helical CT for nephrolithiasis and ureterolithiasis: comparison of conventional and reduced radiation-dose techniques. Radiology 2003;229:575-80, doi:10.1148/radiol. 2292021261.

16. Smith R, Verga M, McCarthy S, et al. Diagnosis of acute flank pain: value of unenhanced helical CT. $A 7 R A m \mathcal{F}$ Roentgenol 1996;166:97-101. 